\title{
Amitriptyline overdose complicated by intestinal pseudo-obstruction and caecal perforation
}

\author{
Andrew J. McMahon \\ Department of General Surgery, Southern General Hospital, Glasgow G51, UK.
}

\begin{abstract}
Summary: I report a case of intestinal pseudo-obstruction, spontaneous caecal perforation and faecal peritonitis caused by an overdose of amitriptyline. This rare but serious complication should be borne in mind in patients who remain constipated while convalescing after self-poisoning with tricyclic antidepressants.
\end{abstract}

\section{Introduction}

Self-poisoning accounts for a significant proportion of acute medical admissions ${ }^{1}$ and tricyclic antidepressant (TCA) overdoses account for $20 \%$ of severe cases of self-poisoning. ${ }^{2}$ The major complications are coma, convulsions, and cardiac arrhythmias. ${ }^{3}$ I report a previously unreported complication - intestinal pseudo-obstruction and spontaneous caecal perforation.

\section{Case report}

A 44 year old man, with longstanding congenital hydrocephalus, took an overdose of $750 \mathrm{mg}$ amitriptyline. He had been taking $75 \mathrm{mg}$ amitriptyline daily over several months for depression, without any side effects. On admission, 16 hours after the overdose, he was drowsy but orientated. Blood pressure was normal and his pulse rate was $110 /$ minute, with the electrocardiogram showing only a sinus tachycardia. He had increased reflexes in his arms and legs related to his longstanding hydrocephalus. There was no past history of altered bowel habit. Serum electrolytes were normal. Apart from urinary retention requiring temporary catheterization, his recovery over the first week was uneventful.

However, he became constipated and had increasing abdominal distension. On the eleventh day, he developed bilious vomiting and watery diarrhoea, but denied abdominal pain. On examination, the pulse rate was 120 /minute, the blood pressure normal and the temperature $38^{\circ} \mathrm{C}$. The abdomen was grossly distended and tympanitic but nontender. Abdominal

Correspondence: A.J. McMahon, M.B., Ch.B., 42 Airlie Street, Hyndland, Glasgow G12 9TR, UK.

Accepted: 28 April 1989
X-ray showed massive dilatation of the whole of the large bowel, some loops of small bowel and a large pneumoperitoneum (Figure 1). At laparotomy, a large caecal perforation and a pelvic collection of pus was found but no mechanical cause for the massive colonic dilatation was found. The large bowel, which contained only air and faecal fluid, was decompressed through a caecostomy tube, and the caecal perforation oversown. Postoperatively, he received intravenous antibiotics and he made an uneventful recovery, with

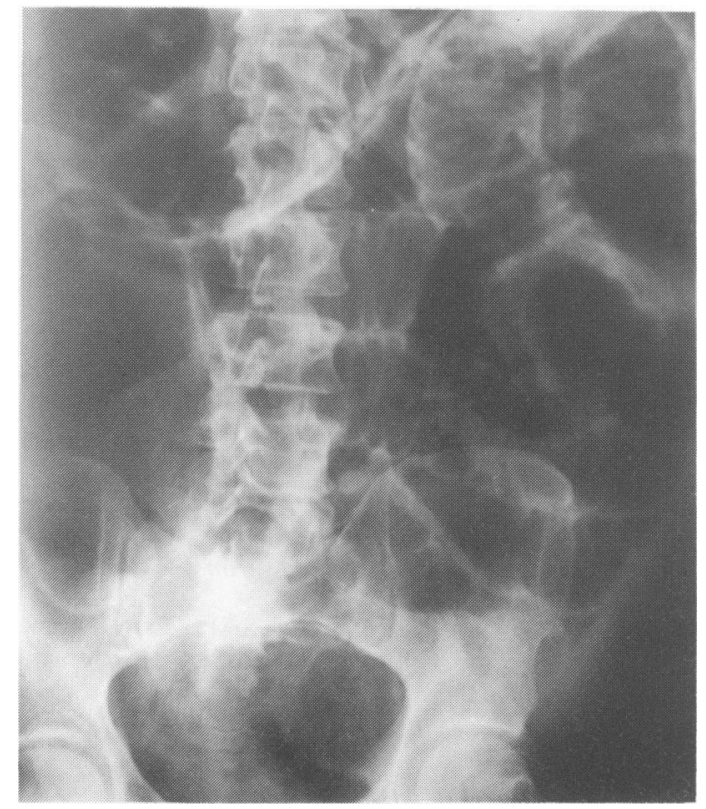

Figure 1 Supine plain abdominal X-ray showing massive colonic dilatation, small bowel dilatation, and a large pneumoperitoneum.

The Fellowship of Postgraduate Medicine, 1989 
return of a normal bowel movement. The caecostomy tube was removed at 10 days.

\section{Discussion}

Acute intestinal pseudo-obstruction is a fairly common transient reversible illness, which mimics mechanical large bowel obstruction in presentation. There is a myriad of associated causes which have been well summarized and reviewed. ${ }^{4}$ After a mechanical cause of obstruction has been excluded by barium enema, the treatment is conservative, with intravenous fluids, nasogastric suction and colonic decompression with a flatus tube or colonoscopy. ${ }^{5}$ However, if caecal diameter exceeds $12 \mathrm{~cm}$, tube caecostomy is advised to prevent caecal perforation. ${ }^{6}$

TCA have anti-cholinergic properties which result in constipation in up to $60 \%$ of treated patients. ${ }^{7}$ There are several reports of TCA causing chronic constipation leading eventually to faecal impaction and intestinal obstruction. ${ }^{8-11}$ Most of the patients have been psychiatric in-patients, who have, in addi-

\section{References}

1. Proudfoot, A.T. \& Pack, J. Changing pattern of drugs used for self-poisoning. Br Med J 1978, 1: 90-93.

2. Strom, J., Madsen, P.S., Nielsen, N.N. \& Sorensen, M.B. Acute self-poisoning with tricyclic antidepressants in 295 consecutive patients treated in an ICU. Acta Anaesthesiol Scand 1984, 28: 666-670.

3. Strom, J., Madsen, P.S., Nielsen, N.N. \& Sorensen, M.B. Acute self-poisoning with tricyclic antidepressants in 295 consecutive patients treated in an ICU. Acta Anaesthesiol Scand 1984, 28: 666-670.

4. Anuras, S. \& Baker, C.R. The colon in the pseudoobstructive syndrome. Clin Gastroenterol 1986, 15: 745-762.

5. Groff, W. Colonoscopic decompression and intubation of the cecum for Ogilvie's syndrome. Dis Colon Rectum 1983, 26: 503-506.

6. Attiyeh, F.F. \& Knapper, W.H. Pseudo-obstruction of the colon (Ogilvie's syndrome). Dis Colon Rectum 1980, 23: $106-108$. tion, been taking large doses of phenothiazines. ${ }^{8-10}$ By contrast, there are only two clearly documented cases of acute intestinal pseudo-obstruction which have been attributed solely to TCA: ${ }^{12}$ the first case was a 14 year old girl with cerebral palsy and the second case was an 81 year old psychiatric in-patient. It is likely that in the former case and also in the current case report, the pre-existing neurological disease predisposed to the occurrence of intestinal pseudoobstruction. There are only two other reported cases associated with TCA: ${ }^{6,13}$ in the first ${ }^{6}$ the patient was taking a large dose of a phenothiazine concomitantly, and in the second case, ${ }^{13}$ compression fractures of the upper lumbar vertebrae were the precipitating cause of the pseudo-obstruction. There have been no reported cases occurring after a TCA overdose. In a recently reported series of nearly 300 severe TCA overdoses, no gastrointestinal complications were noted. ${ }^{3}$

Intestinal pseudo-obstruction is a rare complication of TCA overdose and is more likely to ensue if the patient is elderly and there is concomitant neurological disease.

7. Ayd, F.J. Amitriptyline (Elavil) therapy for depressive reactions. Psychosomatics 1960, 1: 1.

8. Burkitt, E.A. \& Sutcliffe, C.K. Ileus after amitriptyline. Br Med J 1961, 2: 1648.

9. Warnes, H., Lehmann, H.E. \& Ban, T.A. Adynamic ileus during psychoactive medication: a report of three fatal and five severe cases. Can Med Assoc J 1967, 96: 1112-1113.

10. Milner, G. \& Buckler, E.G. Adynamic ileus and amitriptyline: three case reports. Med J Aust 1964, 1: 921-292.

11. Gander, D.R. \& Devlin, H.B. Ileus after amitriptyline. Br Med J 1963, 1: 1160.

12. Milner, G. \& Hills, N.F. Adynamic ileus and nortriptyline. $\operatorname{Br}$ Med J 1966, 1: 841-842.

13. Fletcher, J.P. \& Little, J.M. Intestinal pseudoobstruction. Med J Aust 1979, 2: 339-341. 